\title{
COVID-I9: A IMPORTÂNCIA DO AUTOCUIDADO APOIADO A PACIENTES COM DIABETES TIPO II, FAVORECIDO PALA EQUIPE DE APOIO MATRICIAL NASF-AB
}

\section{COVID-I9: THE IMPORTANCE OF SELF-CARE SUPPORTED TO PATIENTS WITH TYPE II DIABETES, FAVORED BY THE NASF-AB MATRIX SUPPORT TEAM}

\author{
Sandra Patrícia de Oliveira Vilaça. ${ }^{1}$ \\ Diógenes José Gusmão Coutinho
}

\begin{abstract}
RESUMO - Esse estudo objetivou discutir a aprendizagem como um indicador para o manejo do controle das complicações do diabetes tipo 2, assim como seu autocuidado durante a pandemia do novo coronavírus (SARS-CoV-2), causador da COVID-19. Durante a vivência dos profissionais da equipe de matriciamento do Núcleo Ampliado da Saúde da Família na Atenção Básica (NASF-AB) nos atendimentos domiciliares e teleatendimento, observou-se que muito tem informações sobre sua condição de saúde, mas muitos não aderiam ao tratamento. Tratouse de um estudo transversal, com abordagem qualitativa, e etnográfica, com a pergunta norteadora "o que significa o autocuidado para você?" Como resultado que o cuidado apoiado serve para organizar o pensamento; torna-se um espaço mobilizador de processos de interação, que favorece à aprendizagem e a discussão de situações desafiadoras para o enfrentamento da doença com maior aporte de conhecimentos e com a construção de um sujeito ativo e autônomo do cuidado.
\end{abstract}

Palavras-chave: Autocuidado. Cuidado apoiado. Diabetes mellito. Novo coronavírus.

ABSTRACT: This study aimed to discuss learning as an indicator for the management of the control of type 2 diabetes complications, as well os its self-care during the pandemic of the new coronavirus (SARS-CoV-2), which causes COVID-19. During the experience of the professionals of the matrix team of the Extended Nucleus of Family Health in Primary Care (NASF-AB) in home care and call center, it was observed that much has information about their health condition, but many did not adhere to the treatment. It was a cross-sectional study, with a qualitative and ethnographic approach, with the guiding question "what does self-care mean to you?"As a result, supported care serves to organize thinking; it becomes a space that mobilizes interaction processes, which favors learning and the discussion of challenging situations for coping with the disease with greater knowledge and with the construction of an active and autonomous subject of care.

Keywords: Self-care. Supported care. Diabetes mellito. New coronavirus.

\footnotetext{
I Graduada em Nutrição, aluna de Especialização em Pesquisa Avançada pela Faculdade Alpha, mestrado Internacional pela Atenas College University. E-mail: sp.vil.aca@hotmail.com.

2 Graduado em Biologia pela UFPE. Doutor em Biologia pela Universidade Federal de PernambucoUFPE. E-mail: alphadiogenes@gmail.com.
} 


\section{INTRODUÇÃO}

Diabetes mellitus (DM) é um problema de saúde pública, o aumento da sua prevalência está associado a diversos fatores, como: rápida urbanização, transição epidemiológica, transição nutricional, maior frequência de estilo de vida sedentário, maior frequência de excesso de peso, crescimento e envelhecimento populacional, e à maior sobrevida dos indivíduos com diabetes, vem associado a maior taxa de hospitalização, e utilização de serviços de saúde, bem como uma maior incidência de doenças cardiovasculares e cerebrovasculares, cegueira, insuficiência renal e amputações não traumáticas de membros inferiores (DIRETRIZ, 2018)

A pandemia relacionada ao novo coronavírus (SARS-CoV-2), causador da COVID-19, vem impactando negativamente na saúde da população e na economia mundial, dada a inexistência de profilaxia, tratamento específico e sua alta taxa de transmissibilidade, o Brasil decretou Emergência de Saúde Pública de Importância Nacional e, seguindo recomendações e experiências internacionais, adotou o isolamento social como a principal medida de prevenção e controle da doença (FILHO. 2020). Atualmente, sabe-se que dos casos da doença, 81\% serão leves, I4\% graves e 5\% críticos. Os riscos parecem aumentar em pessoas com mais de 70 anos, particularmente aquelas com cardiopatia, diabetes ou doenças respiratórias (Wu e McGoogan, 2020)

A capacidade do sistema de saúde de desempenhar plenamente suas funções no contexto da pandemia demanda não apenas expandir o número de leitos hospitalares e de UTI, mas também reorganizar os fluxos na rede de atendimento, redefinir os papéis das diferentes unidades e níveis de atenção e criar pontos de acesso ao sistema de saúde, especialmente por via remota. Um plano com protocolos a serem seguidos por todos os níveis de atenção, incluindo normas de proteção aos trabalhadores e cuidados para evitar a disseminação do SARS-CoV-2 nas unidades de saúde é fundamental para articular todas essas ações. (DUNAS, 2020)

Em 2013, a Pesquisa Nacional de Saúde (PNS), realizada pelo Instituto Brasileiro de Geografia e Estatística (IBGE) e pelo Ministério da Saúde, estimou que 6,2\% da população brasileira com 18 anos ou mais referiu diagnóstico médico de diabetes, sendo de 7,0\% nas mulheres e de 5,4\% nos homens. (PNS, 2013) O impacto para o indivíduo e sua família como dor, ansiedade, inconveniência e perda da qualidade de vida é difícil quantificar.

O senso comum é que motivar as pessoas a assumirem comportamentos saudáveis é uma tarefa árdua e frustrante, e o processo de mudança, em contrapartida, exige a elaboração de um plano de cuidado com metas pactuadas, monitoramento periódico, habilidade em resolver problemas e prevenção de deslizes e recaídas. (CAVALCANTI, 20I2)

As equipes do Núcleo Ampliado de Saúde da Família e Atenção Básica (NASF-AB) 
constituem um importante corpo de profissionais no âmbito da Atenção Primária à Saúde (APS). No atual cenário de pandemia pelo novo coronavírus (COVID-ı), a atuação de tais profissionais é de relevância ainda maior no que tange ao cuidado coletivo, ao cuidado individual e familiar e também na coordenação e continuidade do cuidado. Entre as ações em apoio ao trabalho das equipes está no suporte e monitoramento de grupos vulneráveis e de risco ao novo coronavírus, que necessitarão de atenção especial no contexto da pandemia por sua situação de saúde (portadores diabetes, hipertensão e outras condições crônicas, gestantes, puérperas, idosos, pessoas vítimas de violência, etc.) e/ou vulnerabilidade social, articulando-se a iniciativas comunitárias e outros setores de políticas públicas, caso necessário, para esses casos. É importante a garantia do acompanhamento desse público nos casos graves, cuja interrupção do cuidado ofereça risco à saúde (NOTA TÉCNICA SAPS/SGAIS/SES-RJ n. o oI/2020)

No caso dos indivíduos portadores de diabetes Mellitus tipo 2, por exemplo, o objetivo é prevenir complicações da doença, aprendendo a viver com a doença, desenvolvendo sua autonomia. Para isso, tarefas como atividade podem englobar mudanças nos hábitos alimentares, como questões sobre o manejo da dieta; o conhecimento, detecção e condutas frente aos sinais e sintomas de hiperglicemia e hipoglicemia; o manejo no preparo, controle e administração de insulina; noções de higiene e cuidado corporal; maior adesão ao uso de medicamentos prescritos, entre outras, assim oportuniza o aprendizado para o autocuidado, favorece uma maior adesão ao tratamento e aumenta o vínculo com a unidade de saúde, através da integração e a união de interesses e motivações. (BRASIL, 2006)

Além do teleatendimento, a consulta domiciliar pelas equipes do Núcleo Ampliado de Saúde da Família e Atenção Básica (NASF-AB) pode garantir a manutenção da assistência a pacientes de maior complexidade e risco, respeitando o distanciamento social, possibilitam o acompanhamento de pacientes sem telefone, evitando, assim, as visitas desnecessárias à UBS.

A vivência dos profissionais com os portadores de diabetes Melittus tipo 2, observou-se que o vírus trouxe consigo o medo, que é um poderoso motivador que pode acelerar a mudança para o bem-estar preventivo. Ele colocou uma perspectiva diferente sobre a real importância do autocuidado, porque as pessoas nos grupos de risco da COVID-ig são aquelas com co-morbidades ou condições de saúde pré-existentes, defendemos que, em tempos de pandemia, é fundamental a humanização do cuidado, o diálogo e as relações de confiança entre profissionais e usuários, então pretende-se com este artigo discutir a aprendizagem como um indicador para o manejo do seu controle. 


\section{METODOLOGIA}

Trata-se de um estudo tipo transversal com abordagem qualitativa, etnográfico, têm como ponto principal entender, descrever e, algumas vezes, explicar, os fenômenos sociais e culturais de grupos sociais e/ ou indivíduos. Foi realizado com pessoas devidamente cadastradas na unidade de saúde da família do Bairro Novo I, situado no município de Camaragibe-PE, a unidade de saúde já tem o levantamento de todos os diabéticos que estão em cadastros individuais e domiciliares pelo relatório operacional de registro eletrônico do e-SUS-AB e registro do controle manual dos agentes comunitários de saúde, onde foi o campo de pesquisa, que participaram do teleatendimento ou atendimento domiciliar da equipe de apoio matricial do Núcleo Ampliado de Saúde da Família na Atenção Básica (NASF-AB), com diagnóstico de diabetes mellitus tipo 2

Os critérios de inclusão foram: portadores de diabetes mellitus do tipo 2, com idade mínima de 18 anos, capazes de responder verbalmente. A coleta de dados em etnografia pressupõe a convivência do pesquisador com o grupo em estudo, no seu ambiente cultural, por um determinado período, com a finalidade de ampliar a escuta do mesmo, a observação e a participação em seu mundo. Neste sentido, o pesquisador é o principal instrumento para a coleta de dados, utilizando como métodos fundamentais para a coleta dos dados a entrevista e observação. O trabalho de campo constituiu-se das seguintes etapas: aproximação com os grupos, coleta de dados por meio de entrevista semiestruturada e observação participante, realizada no período de março a outubro de 2020 .

A observação participante desenvolveu-se durante os teleatendimento e consultas domiciliares, sendo esclarecida, aos participantes, a presença da pesquisadora (primeira autora deste estudo) ao iniciar o trabalho de campo. Esses atendimentos aconteciam diariamente, na própria UBS (por teleatendimento) ou no domicilio, os atendimentos domiciliares aconteciam a partir de um cronograma previamente definido e acordado entre todos, com a participação de cerca de quinze pessoas. Ao término dos atendimentos, as observações foram anotadas em um diário de campo, com registro e descrição detalhados das impressões, notas de campo e notas pessoais. Ao todo, foram observados 15 portadores de diabetes tipo 2. O local das entrevistas foi no consultório da UBS, ou nos domicílios visitados. Antes de iniciar a entrevista, os informantes foram comunicados quanto aos objetivos da pesquisa a partir da leitura do termo de consentimento livre e esclarecido, sendo orientados sobre a necessidade de usarem nomes fictícios, para preservação do anonimato.

Ao todo, foram realizadas quinze entrevistas, a partir da seguinte pergunta descritiva, considerada como questão norteadora: "Fale para mim o que significa o autocuidado para você?" 


\section{FUNDAMENTAÇÃO TEÓRICA}

Autocuidado significa deixar de ser passivo em relação aos cuidados e diretrizes apontadas pela medicina. Trata-se de um comportamento pessoal, que pode influenciar na saúde, porém não se dá de maneira isolada, mas em conjunto com fatores ambientais, sociais, econômicos, hereditários e relacionados aos serviços da saúde. (GOMIDES, 2013)

De acordo com o American Diabetes Associationı, Diabetes Mellitus tipo 2 (DM2) é um grupo de alterações metabólicas caracterizadas pela presença de hiperglicemia, que pode ser ocasionada pela falha na secreção e/ou resistência insulínica, hormônio responsável pelo metabolismo glicídico. Hábitos alimentares inadequados, aliados a níveis de sedentarismo e, consequentemente, ao sobrepeso e à obesidade predispõem a resistência à insulina, síndrome metabólica e DM2. (DANIELLE, 2014)

Dentre as políticas públicas para o DM e os consensos internacionais, um dos atributos em destaque dos profissionais da saúde, em especial, os que compõem a Atenção Primária à Saúde (APS) é o desenvolvimento de atividades educativas, tanto no âmbito individual como coletivo para as pessoas com DM.2. Com abordagem sociocultural, em que o ser humano é compreendido em seu contexto; é sujeito de sua própria formação e se desenvolve por meio de um profícuo processo de ação-reflexão-ação; capacita as pessoas a aprenderem, evidenciando a necessidade de ação concreta com base e valorização da sua realidade social, visando situações limites e superação das contradições. (SALCI, 2018)

Diversos estudos discutem a baixa adesão às atividades de autocuidado com o diabetes, descrevendo possíveis fatores responsáveis pela ascensão dessa problemática. Fatores de ordem pessoal, socioeconômica e cultural, além de aspectos relativos à doença, ao tratamento, ao sistema de saúde e à equipe multiprofissional podem influenciar o autogerenciamento dos cuidados. (NETA, 2015)

A doença resultante da infeção pelo novo coronavírus SARS-COV-2 e designada COVID-ı9 pela OMS foi identificada pela primeira vez em humanos em dezembro de 2019, na cidade de Wuhan, na China. No dia 30 de janeiro de 2020, a OMS declarou estado de Emergência em Saúde Pública em âmbito Internacional e no dia II de março foi declarada pandemia. Os sintomas e o curso da infeção foram inicialmente considerados semelhantes aos da gripe, embora pudessem evoluir para uma infecção respiratória aguda grave com pneumonia e exigir cuidados intensivos para entre I a $5 \%$ dos contaminados (RUSSELL et al., 2020). Com a deflagração da epidemia o governo intensificou uma série de medidas, entre elas a recomendação de quarentena e isolamento social, e no campo da saúde, faz-se necessário, ações em dois âmbitos: (I) macropolítico, principalmente a aquisição e garantia de leitos hospitalares, respiradores e 
medidas farmacológicas; e (2) práticas educativas e ações de cuidado pautadas nas tecnologias leves.

Com a ruptura do tecido social e as novas formas de viver da sociedade, torna-se evidente a temática do cuidado apoiado pelos profissionais de saúde da rede primária de atenção (APS) e apoiadores do NASF-AB, para pacientes diabéticos, exercício cotidiano de ações de educação em saúde, visando produzir sentido na população quanto às orientações impostas por decretos macropolítico. Assim, apostar-se-ia no diálogo como ferramenta imprescindível durante a pandemia, no qual o profissional de saúde, a partir da compreensão da realidade na qual o sujeito está inserido, contribuiria na construção dos sentidos necessários à prática da quarentena, do isolamento social e de outras medidas de prevenção. (CECONN, 2020)

\section{RESULTADOS E DISCUSSÃO}

Destaca-se a presença da ressignificação, da possibilidade, do encontro de soluções, da construção de rede de cuidado e do fortalecimento do autocuidado e da autonomia: Aqui aprendemos como cuidar melhor da nossa saúde, que não estamos só nessa luta, mas quem cuida de nós, somos nós mesmos. (paciente)

Acho que estou experto em relação ao que sentimos, e aprendemos a importância de fazermos melhores escolhas para nossa vida. (paciente)

Aprendemos com as nossas próprias experiências pessoais, somos o autor da nossa aprendizagem. (paciente)

Estou tomando mais água, estou cuidado melhor da minha alimentação (paciente)

Aqui fui bem acolhida, pude tirar minhas dúvidas, das coisas que poderia comer (paciente)

As ações de educação e saúde com promoção do autocuidado apoiado pelos profissionais de saúde, já existiam nas unidades, antes da crise sanitária, no entanto, eram realizadas sempre de forma presencial, o plano de cuidado a pacientes diabéticos, foi estabelecido para evitar a descontinuidade do cuidado e consequentemente agravamento das condições de saúde préexistentes.

Para Mendes e Viana, citados por Ruiz-Moreno et al. (2005, p. 195), a "educação influencia e é influenciada pelas condições de saúde, estabelecendo um estreito contato com todos os movimentos de inserção nas situações cotidianas em seus complexos aspectos sociais, políticos, econômicos, culturais, dentre outros. Educação popular em saúde, que reconhece que os saberes são construídos diferentemente e, por meio da interação entre sujeitos, esses saberes se tornam comuns ao serem compartilhados (GONÇALVES et al., 2008). 
Nesse complexo processo de ensino-aprendizagem, não se pode perder de vista o objetivo da ação educativa: desenvolver a personalidade integral do aprendiz, sua capacidade de pensar e raciocinar, seus valores e hábitos de responsabilidade e cooperação (BORDENAVE, 1994). Essas pessoas compreendem que é possível aprender o cuidado com a saúde, principalmente quando são valorizados e respeitados pela sua experiência de vida, tornando possível uma maior qualidade de vida.

Cada participante compreende que o autocuidado é imprescindível para o controle das co-morbidades, principalmente em período de pandemia de covid-19 em curso, onde o medo do adoecimento é visível, e os problemas emocionais que interferem com a saúde a presença dos profissionais no teleatendimento e em seus domicílios contribui para esclarecer dúvidas e trocas de informações, a comunicação, que para (FREIRE, 2005) "é o indispensável caminho nas questões vitais e em todos os sentidos do nosso ser".

\section{CONSIDERAÇÕES FINAIS}

Atividades educativas, além de possibilitar uma visão crítica e uma maior participação e autonomia das pessoas para enfrentarem as múltiplas facetas vivenciadas em seus processos saúde/doença, deve buscar a corresponsabilização do cuidado integral, todos os profissionais da equipe de saúde tem um papel importante no apoio ao autocuidado e na qualificação da atenção

de seus usuários. É necessário somar saberes para dar respostas efetivas e eficazes aos problemas complexos que envolvem a perspectiva de viver com qualidade. É preciso também considerar todos os atores sociais envolvidos, valorizando todos os saberes presentes no cenário do território, tendo como centro as necessidades apresentadas pelos usuários

Torna-se eficaz o aconselhamento das medidas não farmacológicas e farmacológicas apoiado, que reduzem o risco das co-morbidades, inserindo algum nível de atividade física na rotina e dando dicas para otimizar a dieta com base nos alimentos saudáveis.

\section{REFERÊNCIAS BIBLIOGRÁFICAS}

BRASIL. Ministério da Saúde. Secretaria de atenção à saúde. Protocolo Do Manejo Clínico Do Coronavírus (COVID-ıg) Na Atenção Primária. Secretaria de Atenção Primária à Saúde (SAPS), 2020 . disponível em: $<$ https://portalarquivos2.saude.gov.br/images/pdf/2020/marco/24/20200323-ProtocoloManejovero5.pdf

BRASIL. Ministério da Saúde. Secretaria de Atenção à Saúde. Departamento de Atenção Básica. Diabetes Mellitus / Ministério da Saúde, Secretaria de Atenção à Saúde, Departamento de 
Atenção Básica. - Brasília : Ministério da Saúde, 2006 disponível em:< http://bvsms.saude.gov.br/bvs/publicacoes/diabetes_mellitus.PDF

BORDENAVE, J. D. Alguns fatores pedagógicos. [Apostila do curso de capacitação pedagógica para instrutor/ supervisor da área da saúde - Ministério da Saúde. Coordenação Geral de Desenvolvimento de Recursos Humanos para o SUS]. Brasília, 1983 disponível em:< https://www.nescon.medicina.ufmg.br/biblioteca/imagem/o22o.pdf

CAVAlCANTI, Ana Maria; Oliveira, ÃNGELA Cristina Lucas de. Autocuidado Apoiado: manual do profissional de saúde/organização, Curitiba: Secretaria Municipal de Saúde, 2012. 92p. Disponível em:< file:///C:/Users/Nagem/Downloads/Autocuidado-Apoiado.pdf

CECCON, Roger Flores; SCNEIDER, Ione Jayce Ceola. Tecnologias leves e educação em saúde no enfrentamento à pandemia da COVID-rg. disponível em:< file://C:/Users/Nagem/Downloads/136-Preprint\%20Text-I46-I-Io-20200422\%20(5).pdf

DANIELE, Thiago Meideiros da Costa; VASCONCELOS, João Paulo; COLTINHO, Francisco Girleudo. Avaliação do autocuidado de pacientes com Diabetes Mellitus tipo 2 em uma unidade de atenção básica: Cinergis 2014;15(3):135-139 Disponível em:< https://online.unisc.br/seer/index.php/cinergis/article/view/499o/3893

DAUNAS, Regina Paiva; SILVA. Gilmar Azevedo e; TASCA, Renato; LEITE, Iure da costa; BRASIL, Patrícia; GRECO, Dirceu B; GRABOIS, Victor; CAMPOS, Gastão Vagner. O papel da atenção primária na rede de atenção à saúde no Brasil: limites e possibilidades no Cad. Saúde Pública 36 (6) 26 Jun 2020202oenfrentamento da COVID-ig. Disponível em:< https://www.scielosp.org/article/csp/2020.v36n6/eooro4120/

Diretrizes da Sociedade Brasileira de Diabetes 2017-2018 / Organização José Egídio Paulo de Oliveira, Renan Magalhães Montenegro Junior, Sérgio Vencio. -- São Paulo: Editora Clannad, 2017. Disponível em: https://www.diabetes.org.br/profissionais/images/2017/diretrizes/diretrizes-sbd-2017-2018.pdf FILHO, Breno Augusto; TRITANY, Érika Fernandes. COVID-ı: importância das novas tecnologias para a prática de atividades físicas como estratégia de saúde pública. Cad. Saúde Pública vol.36 no.5 Rio de Janeiro 2020 Epub May 18, 2020. disponível em:< https://www.scielo.br/scielo.php?script=sci_arttext\&pid=Soro2-311 X2020000500505

FREIRE P. Educação como prática da liberdade. 28ae ed. São Paulo: Paz e Terra; 2005. Disponível 
em:<https://ipco.org.br/paulo-freire-educacao-como-pratica-da-libertacao/

GOMIDES, Danielle dos Santos; VILlAS-BOAS, Cristiane Gomes; COELHO, Anna CLÁUDIA Martins; PACE; Ana Emília. Autocuidado das pessoas com diabetes mellitus que possuem complicações em membros inferiores: Acta Paul Enferm. 2013; 26(3):289-93. Disponível em:< http://www.scielo.br/pdf/ape/v26n3/r4.pdf

GONÇALVES, M. C. et al. Educação permanente em saúde: dispositivo para a qualificação da Estratégia Saúde da Família. Belém: UFPA, 2008 . disponível em:< https://www.unasus.unifesp.br/biblioteca_virtual/esf/2/unidades_conteudos/unidadeog/unid adeo9.pdf.

IBGE. Pesquisa Nacional de Saúde: 2013 : indicadores de saúde e mercado de trabalho : Brasil e grandes regiões / IBGE, Coordenação de Trabalho e Rendimento. - Rio de Janeiro : IBGE, 2016. 66 p disponível em:< https://biblioteca.ibge.gov.br/visualizacao/livros/liv97329.pdf

MELLO, Filho J. Grupoterapia com pacientes somáticos. In: Osório LC. disponível em:< http://pepsic.bvsalud.org/scielo.php?script=sci_arttext\&pid=SI677-29702005000200004

NETA, Dinah Sá Rezende; SILVA, Ana Roberta Vilarouca da; SILVA, Grazielle Roberta Freitas da. Adesão das pessoas com diabetes mellitus ao autocuidado com os pés. Rev. Bras.Enferm. vol.68 no.r Brasília jan./fev. 2015. disponível em:< https://www.scielo.br/scielo.php?script=sci_arttext\&pid=Soo34-7167201500o100II I\&lng=pt\&tlng=pt

NOTA TÉCNICA SAPS/SGAIS/SES-RJ № or/2020, ORIENTAÇÕES QUANTO À ATUAÇÃO DO NASF-AB NO CONTEXTO DE PANDEMIA COVID-ı, disponível em:< https://coronavirus.rj.gov.br/wp-content/uploads/2020/o6/NOTA-T\%C $3 \% 89$ CNICASAPS-SGAIS-SES-RJ-No-or-2020.pdf RUSSELL, T. W. et al. Estimating the Infection and Case Fatality Ratio for COVID-ig Using Age-Adjusted Data from the Outbreak on the Diamond Princess Cruise Ship. medRxiv, 2020.

RUIZ-MORENO et al. Jornal Vivo: relato de uma experiência de ensino-aprendizagem na área da saúde. disponível em:<https://www.scielo.br/pdf/icse/vgni6/v9ni6azı.pdf 
SALCI, Maria Aparecida; MEIRELlES, Betina Hörner Schlindwein; SILVA, Denise Maria Guerreiro Vieira da. Educação em saúde para prevenção das complicações crônicas do diabetes mellitus na atenção primária. Esc Anna Nery 2018;22(I): e20170262 disponível em:< https://www.scielo.br/pdf/ean/v22nI/pt_1414-8145-ean-2177-9465-EAN-2017-0262.pdf

WU, Z; MCGOOGAN, J. M. Characteristics of and important lessons from the coronavirus disease 2019 (covid-19) outbreak in China: summary of a report of 72,314 cases from the Chinese Center for Disease Control and Prevention. JAMA [Internet]. 2020 Feb [cited 2020 Mar 23]. disponível em:<» https://doi.org/ı.ıoo/jama.2020.2648 